\title{
Rapid Task-Related Plasticity of Spectrotemporal Receptive Fields in the Auditory Midbrain
}

\author{
(1DSean J. Slee and 논 Stephen V. David \\ Oregon Hearing Research Center, Oregon Health and Science University, Portland, Oregon 97239-3098
}

Previous research has demonstrated that auditory cortical neurons can modify their receptive fields when animals engage in auditory detection tasks. We tested for this form of task-related plasticity in the inferior colliculus (IC) of ferrets trained to detect a pure tone target in a sequence of noise distractors that did not overlap in time. During behavior, responses were suppressed at the target tone frequency in approximately half of IC neurons relative to the passive state. This suppression often resulted from a combination of a local tuning change and a global change in overall excitability. Local and global suppression were stronger when the target frequency was aligned to neuronal best frequency. Local suppression in the IC was indistinguishable from that described previously in auditory cortex, while global suppression was unique to the IC. The results demonstrate that engaging in an auditory task can change selectivity for task-relevant features in the midbrain, an area where these effects have not been reported previously.

Key words: attention; auditory; behavior; inferior colliculus; plasticity; STRF

\section{Significance Statement}

Previous studies have demonstrated that the receptive fields of cortical neurons are modified when animals engage in auditory behaviors, a process that is hypothesized to provide the basis for segregating sound sources in an auditory scene. This study demonstrates for the first time that receptive fields of neurons in the midbrain inferior colliculus are also modified during behavior. The magnitude of the tuning changes is similar to previous reports in cortex. These results support a hierarchical model of behaviorally driven sound segregation that begins in the subcortical auditory network.

\section{Introduction}

Humans and other animals have the remarkable ability to attend to one of many competing objects in an auditory scene (Cherry, 1953; Bizley and Cohen, 2013). Psychophysical evidence suggests that selective attention acts on auditory objects, rather than their individual acoustical components, to aid in segregation (ShinnCunningham, 2008). Consistent with this idea, physiological measurements from auditory cortical areas in humans reveal that the neural representation of attended objects is enhanced relative to nonattended objects (Ding and Simon, 2012; Mesgarani and Chang, 2012). Studies in the primary auditory cortex (A1) of animals have shown that neural selectivity is modified during engagement in auditory tasks, often producing changes that en-

Received April 29, 2015; revised Aug. 10, 2015; accepted Aug. 15, 2015.

Author contributions: S.J.S. and S.V.D. designed research; S.J.S. performed research; S.J.S. analyzed data; S.J.S. and S.V.D. wrote the paper.

This work was supported by National Institutes of Health Grants DC010439 (S.V.D.) and DC012124 (S.J.S.). We thank Henry Cooney for technical support and assistance with animal care and Brian Jones for assistance with electrophysiology.

The authors declare no competing financial interests.

Correspondence should be addressed to Sean J. Slee, Oregon Hearing Research Center, Oregon Health and Science University, 3181 SW Sam Jackson Park Road, MC L335A, Portland, OR 97239-3098. E-mail: slee@ohsu.edu.

DOI:10.1523/JNEUROSCI.1671-15.2015

Copyright $\odot 2015$ the authors $\quad 0270-6474 / 15 / 3513090-13 \$ 15.00 / 0$ hance discriminability of task-relevant sounds (Fritz et al., 2003; Otazu et al., 2009; Lee and Middlebrooks, 2011; Niwa et al., 2012; Rodgers and DeWeese, 2014). In secondary cortical belt areas, behavioral modulation is generally stronger and sensory activity shifts from the more veridical representation found in A1 toward an abstract representation of task-related categories and motor signals observed in the frontal cortex (Fritz et al., 2010; Niwa et al., 2013; Atiani et al., 2014). These studies support a model in which the representation of an attended object begins in A1 and emerges gradually as information ascends in the cortical hierarchy.

In mammals, dense corticofugal projections send information from the auditory cortex to subcortical nuclei (Winer, 2006). Thus, neuronal responses in the subcortical auditory system might also be altered during behavior. Consistent with this hypothesis, previous studies using cortical inactivation (Zhang et al., 1997; Nakamoto et al., 2008) and electrical stimulation (Ma and Suga, 2001) have demonstrated a role for these corticofugal projections in long-term plasticity of tuning in the inferior colliculus (IC). A related study found that ablation of corticofugal projections to the IC in ferrets disrupts their ability to adapt to altered spatial cues caused by ear plugging (Bajo et al., 2010). These studies demonstrate that the corticofugal system provides 
one candidate mechanism for plasticity in subcortical nuclei. However, it is unclear how these modulatory signals influence neural activity on the shorter timescales relevant to the behaviors used previously in cortical studies.

The present study tested the hypothesis that spectrotemporal receptive fields (STRFs) of IC neurons are modified when an animal engages in an auditory detection task. The stimuli, animal model, and behavioral paradigm were matched to a previous study in A1 (David et al., 2012), thereby enabling a direct comparison between A1 and the IC. Here we report rapid task-related plasticity of the receptive fields of IC neurons. The frequency specificity, sign, and magnitude of STRF changes at the target frequency are similar to previous reports in A1. However, average evoked responses were consistently suppressed in the IC while changes in average activity were more diverse in $\mathrm{A} 1$. These results support a hierarchical model of auditory attention that begins in the subcortical network.

\section{Materials and Methods}

Surgical procedure. All procedures were approved by the Oregon Health and Science University Institutional Animal Care and Use Committee and conform to the National Institutes of Health standards. Animal care and procedures were similar to those described previously for recording from the auditory cortex of awake behaving ferrets (David et al., 2012). Two young adult male ferrets were obtained from an animal supplier (Marshall Farms). Normal auditory thresholds were confirmed by measuring auditory brainstem responses. A sterile surgery was then performed under isoflurane anesthesia to mount a post for subsequent head fixation and to expose a small portion of the skull for the electrode recording. The head post was surrounded by dental acrylic or Charisma composite, which bonded to the skull and also to a set of stainless-steel screws embedded in the skull. During a 2 week recovery period, the animal was treated daily with antibiotics (Baytril) and the wound was cleaned and bandaged.

Behavioral training. After the ferret recovered from surgery, it was placed on water restriction $18-24 \mathrm{~h}$ before training began. The ferret was gradually habituated to lie still inside a Plexiglas tube with head fixed via a custom stereotaxic apparatus attached to the head post. The ferret was intermittently given water and Nutri-Cal as a reward. Sessions initially lasted for $5 \mathrm{~min}$ and increased by increments of 5-10 min until the ferret lay comfortably for $\geq 1 \mathrm{~h}$. At this time the ferret was placed on a restricted water schedule and auditory behavioral training began.

Water rewards were delivered through a spout 3-4 mm away from the ferret's nose. Water delivery was controlled electronically with a solenoid valve. Licking was monitored by breaking a beam formed by an infrared LED and photodiode placed across the spout. Initially, a $1 \mathrm{~s}$ pure tone of variable frequency ( $60 \mathrm{~dB}$ SPL) was paired with a small water reward. If the animal successfully licked during the presentation of the tone, additional water was delivered. The ferret quickly learned to associate the tone with a water reward. At this point the water reward was delivered only if the ferret licked from 0.2 to $1.2 \mathrm{~s}$ from the onset of the tone.

In the next stage of training, a random number (1-4) of 1 s temporally orthogonal ripple combination (TORC) stimuli (details below) were presented before the tone. The interval between each stimulus was $1 \mathrm{~s}$. A false alarm was recorded if the ferret licked the spout before the target response window and was punished with a $4 \mathrm{~s}$ time out, during which the chamber lights were extinguished. Initially, the TORC stimuli were presented at $20 \mathrm{~dB}$ SPL (a $40 \mathrm{~dB}$ signal-to-noise ratio). The level was gradually increased to $60 \mathrm{~dB}$ SPL (the same level of the tone). Training was complete once the ferret learned to complete this task with an average false alarm rate of $<25 \%$. The entire training procedure took $1-2$ weeks.

Acoustics and stimuli. All behavioral and physiological experiments were conducted inside a custom double-walled sound-isolating chamber (Professional Model, Gretch-Kenn) with inside dimensions of $8 \times 8 \times 6$ feet (length $\times$ width $\times$ height). A custom second wall was added to the single-walled factory chamber by building a wooden frame to which 0.75 inch medium-density fiberboard was attached. The air space between the outer and inner walls was 1.5 inches. Finally, the inside wall was lined with 3 inch sound-absorbing foam (Pinta Acoustics). The chamber attenuated sounds $>2 \mathrm{kHz}$ by $>60 \mathrm{~dB}$. Sounds from 0.2 to $2 \mathrm{kHz}$ were attenuated by $30-60 \mathrm{~dB}$, falling off approximately linearly on a logarithmic plot of level versus frequency.

Sounds were digitally generated in Matlab (Mathworks), digital-toanalog converted (100 kHz; PCI-6229, National Instruments), and presented over a Manger sound transducer (model W05, Manger Audio) driven with a Crown amplifier (model D-75A, Crown Audio). The speaker was placed $1 \mathrm{~m}$ from the animal's head $30^{\circ}$ contralateral to the IC under study. Sounds were calibrated using a 0.5 inch microphone (type 4191, Brüel \& Kjær). All stimuli were presented with $10 \mathrm{~ms} \cos ^{2}$ onset and offset ramps.

The stimuli used in this study were pure tones (sine waves) and TORCs (Klein et al., 2000). TORCs provide an efficient means to characterize a linear approximation of the receptive field of a neuron (see Linear spectrotemporal model, below). TORC stimuli were created by adding ripple stimuli with different spectral and temporal modulation frequencies. The spectral modulation frequencies ranged from -1.4 to 1.4 cycles per octave in steps of 0.2. Temporal amplitude modulation frequencies ranged from 8 to $96 \mathrm{~Hz}$ in steps of $8 \mathrm{~Hz}$. This resulted in sets of 30 TORC stimuli. Four sets of TORC stimuli, each covering five frequency octaves, were created. The ranges were $0.125-4,0.25-8,0.5-16$, and $1-32 \mathrm{kHz}$. During experiments, the set of TORCs was chosen that best spanned the frequency-response range of the neuron under study.

Electrophysiology. At the beginning of the neurophysiology experiments, a small ( $\sim 1 \mathrm{~mm}$ diameter $)$ craniotomy was made in the skull. The location of the hole was based on stereotaxic coordinates as well as superficial landmarks on the skull marked during surgery. The exposed recording chamber surrounding the craniotomy was covered with polysiloxane impression material (GC America) between recording sessions and, after many penetrations (usually $>30$ ), the hole was filled with a layer of bone wax and dental acrylic before another craniotomy was made to provide access to other regions of the IC on the same hemisphere. Multiple craniotomies were performed on both hemispheres before the animal was killed and perfused for histological evaluation.

On each recording day, 1 or 2 tungsten microelectrodes (FHC or A-M Systems, impedance 1-5 M $\Omega$ ) were slowly advanced with independent motorized microdrives (Alpha-Omega). Depending on the angle of the lateral approach to the IC, the electrode traversed 7-9 mm of brain tissue before reaching the IC. The electrode was positioned (Kopf Instruments) approximately in the frontal plane at an angle between $30-50^{\circ}$ medial or lateral and $0-10^{\circ}$ anterior or posterior. Stimulus presentation, animal monitoring via video camera, and electrode advancement were controlled from outside the sound booth. Only well isolated single neurons were studied. Raw neural signals were bandpass filtered $(0.3-10 \mathrm{kHz})$, amplified (10,000×; A-M Systems 1800 or 3600 AC amplifier), digitized (20 kHz; PCI-6052E, National Instruments) and stored on a computer for off-line analysis (details below). Recording sessions were terminated after 3-4 h, or earlier if the animal showed signs of discomfort.

Neurons were isolated using pure tones and/or wideband noise bursts ( $50 \mathrm{~ms}$ duration, $4 \mathrm{~Hz}$ ) of variable level. Recordings were made in both the IC central nucleus (ICC) and regions surrounding the ICC (presumably the lateral and dorsal cortex of the IC). Neurons likely recorded from the ICC were classified as "ICC-like" using the following criteria: (1) they were recorded within the tonotopic map, (2) they had strong responses to pure tones, (3) and they had short latencies consistent with previous studies ( $\sim 5-20 \mathrm{~ms}$ ). Physiological identification of neurons belonging to noncentral divisions of the IC was more challenging. Therefore, all neurons that did not meet these criteria were grouped into a single class labeled the "noncentral inferior colliculus" (NCIC). For a subset of penetrations, we were able to locate recordings to a specific region of the IC (see Histology and track labeling below).

Behavioral analysis. Behavioral performance during each trial of the detection task (2-4 references, one target) was scored as a hit (target response), false alarm (response preceding the target), or a miss (no response). The hit rate was calculated as the number of hits divided by the sum of hits and false alarms. Similarly, the false alarm rate was the number of false alarms divided by the sum of hits and false alarms. The 
discrimination index, $d^{\prime}$, was estimated by $d^{\prime}=$ norminv(hit rate) - norminv(false alarm rate), where norminv is the inverse of the normal cumulative distribution function (Lauer et al., 2011). Hit rates of 1 and false alarm rates of 0 were set to 0.99 and 0.01 , respectively, to avoid infinite values.

Neurophysiological analysis. Putative spikes were extracted from the continuous signal by collecting all events $\geq 4$ SDs from zero. Spikes were detected from the events using principal component analysis and $k$-means clustering (David et al., 2009). Stability of single-unit isolation was verified by examining waveforms and interval histograms. If isolation was lost during a behavioral block, only activity during the stable period was analyzed.

A subset of data published previously from A1 (David et al., 2012) was reanalyzed and compared with the current IC dataset (see Figs. $9,10)$. The A1 dataset consisted of 108 single units and multiunits recorded during the same auditory task when the target frequency was $\leq 0.5$ octaves from the unit's best frequency (BF). For these data, single-unit isolation was quantified from cluster variance and overlap as the fraction of spikes that were likely to be from a single cell rather than from another cell. Only units with $\geq 75 \%$ isolation and $<5 \%$ change in isolation between passive and active conditions were included in the analysis. The BF distributions in the IC and A1 were similar and the median BFs (IC: $6.3 \mathrm{kHz}$; A1: $7.9 \mathrm{kHz}$ ) were not significantly different $(p=0.19$, rank-sum test).

Linear spectrotemporal model. Tuning properties of A1 and IC neurons were characterized by the STRF estimated by reverse correlation between time-varying neuronal spike rate and the rippled noise used as reference sounds during behavior. For a stimulus with spectrogram, $s(x, t)$ (frequency channels, $x=1 \ldots X$, and time, $t=1 \ldots T)$, and instantaneous firing rate $r(t)$, the STRF, $h(x, u)$, is defined as the linear weight matrix that predicts the firing rate response as follows:

$$
r(t)=\sum_{\substack{x=1, u=0}}^{X, U} h(x, u) s(x, t-u)
$$

Each weight of $h$ indicates the gain applied to frequency channel $x$ at time lag $u$ to produce the predicted response. Positive values indicate components of the stimulus correlated with increased firing, and negative values indicate components correlated with decreased firing (see Fig. 3).

Local and global changes during behavior. We investigated both changes in local tuning and global changes in overall excitability during behavior. Figure $1 A$ shows a hypothetical frequency tuning curve of an IC neuron modeled by a Gaussian function (black). During behavior, the animal detects a target frequency, which is typically placed near the BF of the neuron under study (arrow). Local tuning changes during the behavior may result in enhancement (dotted line) or suppression (gray line) of the spike rate in a restricted frequency region around BF (the target frequency). Global gain changes result in a multiplicative scaling of the entire tuning curve (Fig. 1B). Local and global changes can occur in the same neuron. Figure $1 C$ illustrates both global and local suppression while Figure $1 D$ shows local suppression and global enhancement. The following sections describe the methods used to measure these two types of changes during behavior.

Local STRF changes during behavior. Behavior-dependent local STRF changes were determined from the difference between STRF amplitudes estimated during passive listening and behavior. For the analysis of IC neurons (see Fig. 4), the STRF amplitude was measured as the average
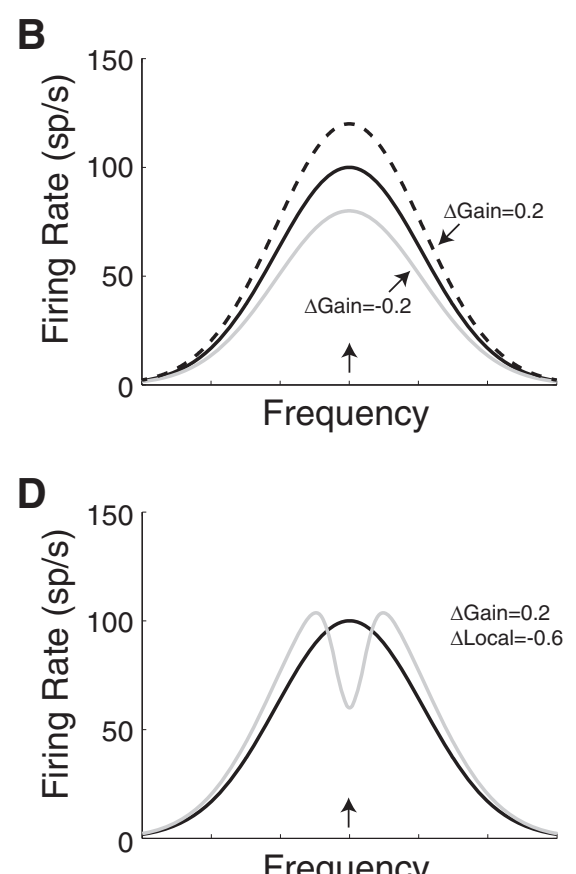

Frequency
Frequency

Figure 1. Models of task-related plasticity. A, Model frequency tuning curve of an IC neuron in the passive condition (black). detection of a target tone at BF (arrow), there is a local tuning change that results in enhancement (dotted line) or ( negative global gain change and a negative local tuning change during behavior (gray line). D, Model response with a positive global gain change and a negative local tuning change during behavior (gray line). sp/s, spikes/second.

weight in a one-octave window centered at the target frequency from 0 to $125 \mathrm{~ms}$ time lag ( 3 frequency bins $\times 25$ time bins $=75$ weights). Only significantly nonzero weights within the window were included in the average ( $p<0.05$ for either passive or active STRF, estimated by jackknifed $t$ test). Significantly nonzero weights at time lags $>50 \mathrm{~ms}$ (10 time bins) were not observed. For the analysis of A1/IC units (see Figs. 9, 10), the STRF amplitude was measured in a one-third-octave window centered at the target frequency at peak delay (one weight). This smaller analysis window was used to match the analysis in the previous A1 study (David et al., 2012). The STRF difference (active - passive) was normalized by the amplitude of the passive STRF. This produced a measure of the local STRF change as a fraction of the passive STRF. The average STRF difference across experiments was the mean across neurons, taken after aligning each STRF difference on the frequency axis at the target frequency for that experiment. Significant local STRF changes were determined by a 20-fold jackknifed $t$ test (Efron and Tibshirani, 1998), which allows for unbiased estimates of SEM for non-Gaussian distributions. For all behavioral analyses, neural responses were compared between passive and correct behavioral trials.

Global gain changes during behavior. Behavior-dependent changes in overall excitability were determined by comparing driven firing rates between behavior conditions. To obtain unbiased measures of global gain changes, average time-varying firing-rate responses to each reference sound were calculated by averaging across repetitions and behavioral conditions ( $5 \mathrm{~ms}$ bins). This resulted in 750 samples of the response rate that were subsequently sorted into ascending order. This sort index was then used to group responses averaged only within each behavior condition (active or passive) in ascending order, with 50 samples in each group (see Fig. 5A). The resulting 15 average active rates were plotted against the corresponding passive rates. Finally, we found the best linear fit to the 15 pairs of rates (by minimizing squared error). The slope of the line was defined as the gain and the intercept as the offset (see Figs. 5, $7-10)$. The global gain change was defined as gain -1 . No change in the driven firing rate during behavior results in a global gain change of zero. 
Significant global gain changes and offsets were tested with 20 -fold jackknifed $t$ tests.

Estimation of the global gain change was the same whether the analysis was performed on driven rates (spontaneous rate subtracted) or the raw firing rates. This was true for all the experimental data reported in this study and in a model with Poisson-distributed spike rates (data not shown). In the model, the offset term was equal to the change in spontaneous rate. In the results we report global gain changes and offsets using the driven rate to dissociate changes in the mean driven rate and spontaneous rate (we also report spontaneous rate changes in Fig. 6). In either case, the offset changes were small and less systematic compared with the global gain changes.

Global gain was used to measure the recovery or persistence of taskrelated plasticity (see Fig. 7). In this analysis, postpassive rates (measured immediately after the behavior) were compared with the prepassive rates (measured immediately before the behavior), using the global gain analysis described above. Full recovery was defined as a global gain change (postpassive relative to prepassive) that was not significantly different from zero. Partially recovered global gain changes had values between the active value and zero. In a subset of neurons, we recorded neural responses during two behaviors: one with the target frequency near $\mathrm{BF}$ and one 1-3 octaves away from BF (Off-BF condition). To compare plasticity between $\mathrm{On}-\mathrm{BF}$ and Off-BF target conditions, global gain changes were computed for the On-BF rates relative to the Off-BF rates (see Fig. 8). Neurons with global gain changes significantly different from zero were considered to have frequency-dependent plasticity. To display the frequency-dependent global gain changes (see Fig. 8), gain for each behavior was recalculated relative to the passive condition.

Local tuning changes during behavior. Local receptive field changes were measured by scaling the passive STRF by the gain before computing the STRF difference at the target frequency (see Figs. 1C,D, 10). The residual STRF difference was defined as a local tuning change (evaluated with a jackknifed $t$ test). A local tuning change of zero indicates that the local STRF change is completely accounted for by the global gain change.

Histology and track labeling. The final track in a subset of the craniotomies was labeled by coating the electrode with a fluorescent lipophilic dye (Vybrant Cell labeling, Invitrogen) as described previously (DiCarlo et al., 1996; Slee and Young, 2013). At the end of all recordings, the animal was killed with barbiturate overdose (Euthasol, $0.5 \mathrm{ml} / \mathrm{kg}$ ) and transcardially perfused ( $0.5 \%$ paraformaldehyde). The brain was sectioned $(50 \mu \mathrm{m})$ in the coronal plane, mounted, and imaged with a $0.5 \times$ objective using bright-field and fluorescence microscopy. In Ferret M, two of two tracks were successfully labeled with fluorescent dye (Fig. 2C, tracks). In Ferret B, zero of six tracks were successfully labeled, possibly due to the long duration between track labeling and perfusion $(>5$ months). Apart from the physiological criteria, tissue damage consistent with electrode tracks near the craniotomy in the same coronal section as the IC was taken as confirmation of successful targeting of the IC in this animal.

\section{Results}

We studied the effects of task engagement on the response properties of single neurons in the IC. Two ferrets (B and M) were trained on a go/no-go task to report pure tone targets and ignore broadband noise reference stimuli (TORCs; Klein et al., 2000; Fig. 2A). The animals were required to withhold licking from a water spout during presentation of the TORCs and were given a water reward for licking after target tone onset. Both animals learned this task after 1-2 weeks of training. Hit rate and false alarm rate were measured during all 129 behavior sessions with simultaneous IC recordings (Fig. $2 B$ ). Both animals performed consistently above chance (B average $d^{\prime}, 2.7$; M average $d^{\prime}, 1.7$ ).

We made recordings in several regions of the IC while the animals performed the task. Successful targeting of the IC was confirmed postmortem by labeling tracks using microelectrodes coated with fluorescent dye (Fig. 2C; see Materials and Methods). We recorded responses from 95 single neurons: 48 in the ICC and
A
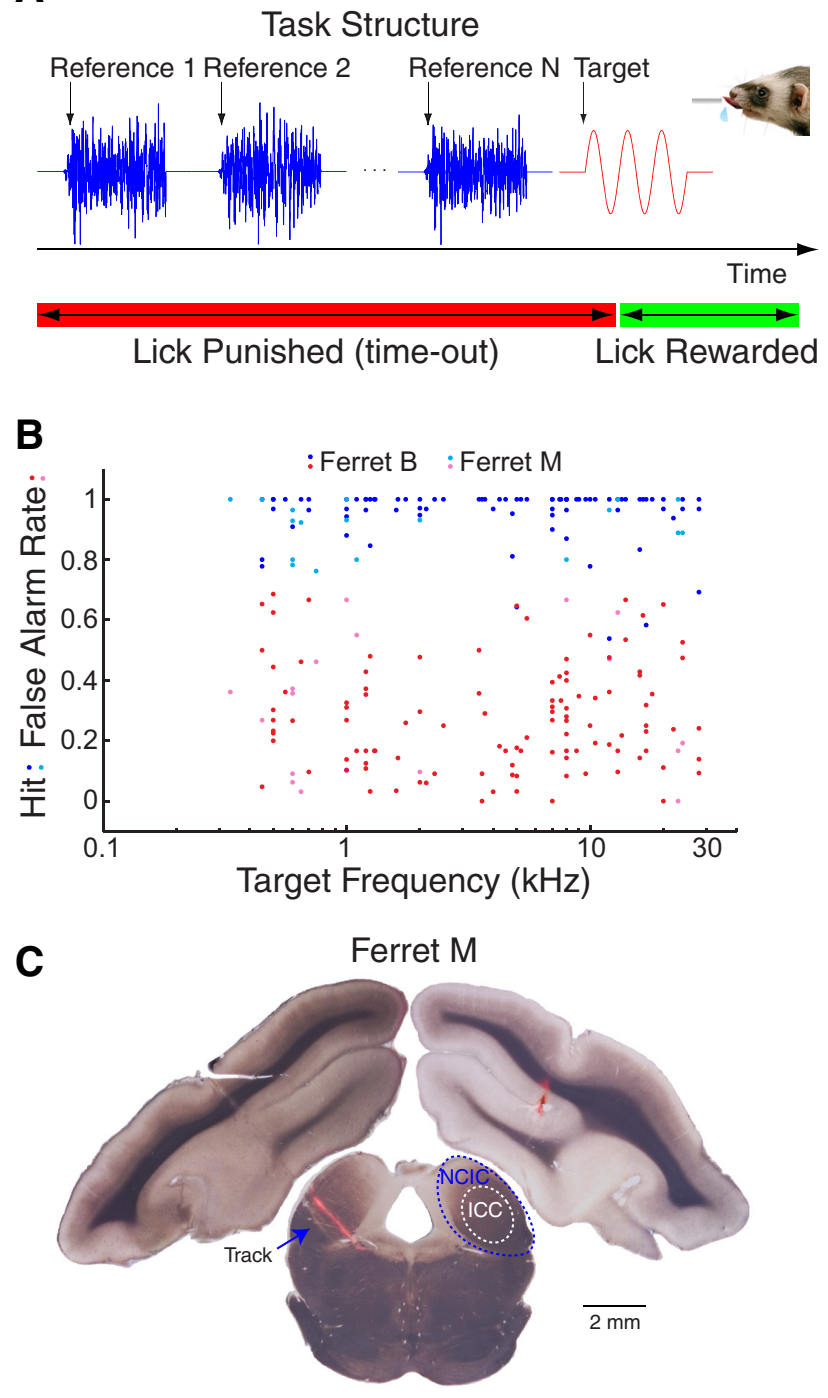

Figure 2. Methods and behavioral results. $A$, During the task, ferrets were required to report a pure tone target (red) following a random number of reference noise sounds (blue). Ferrets were rewarded with water for licking a spout 0.2-1.2 $\mathrm{s}$ after target onset (green bar) and punished with a timeout for licking earlier (red bar). $\boldsymbol{B}$, Hit rate (blue, cyan) and false alarm rate (red, magenta) are plotted against target frequency for all behavior sessions in two ferrets ( $B$ and $M$ ) during neural recordings in the IC. (Ferret B performed in 111 sessions and Ferret $M$ performed in 18). Since the BF often matched the target frequency ( 95 of 129 sessions), this plot also shows the range of BFs sampled in the IC. C, Coronal section of the brain of Ferret $M$ at the level of the IC. The arrow marks a fluorescently labeled track that passed through the left IC. Dotted lines on the right IC indicate the approximate borders between the ICC and NCIC divisions of the IC.

47 from regions outside the ICC, which were combined into a group labeled NCIC (see Materials and Methods).

\section{STRFs are suppressed at the target frequency during the auditory task}

To test for effects of task engagement on neuronal selectivity, we compared STRFs measured from responses to the TORC stimuli in the behaving and passive conditions. The STRF for an example ICC neuron measured during passive listening reveals excitatory tuning to a narrow range of frequencies around a $\mathrm{BF}$ of $1 \mathrm{kHz}$ with a latency of $10 \mathrm{~ms}$ (Fig. 3Ai). During behavior, the target frequency was fixed near the BF at $0.75 \mathrm{kHz}$. The STRF of the same neuron measured while the ferret was engaged in the detec- 
A ICC: mgc16eCh2

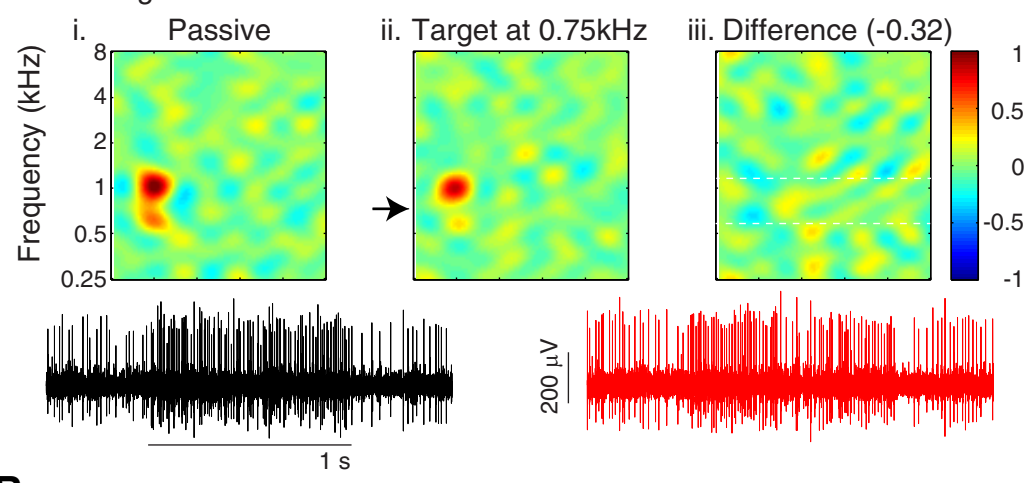

B NCIC: btn37aCh2
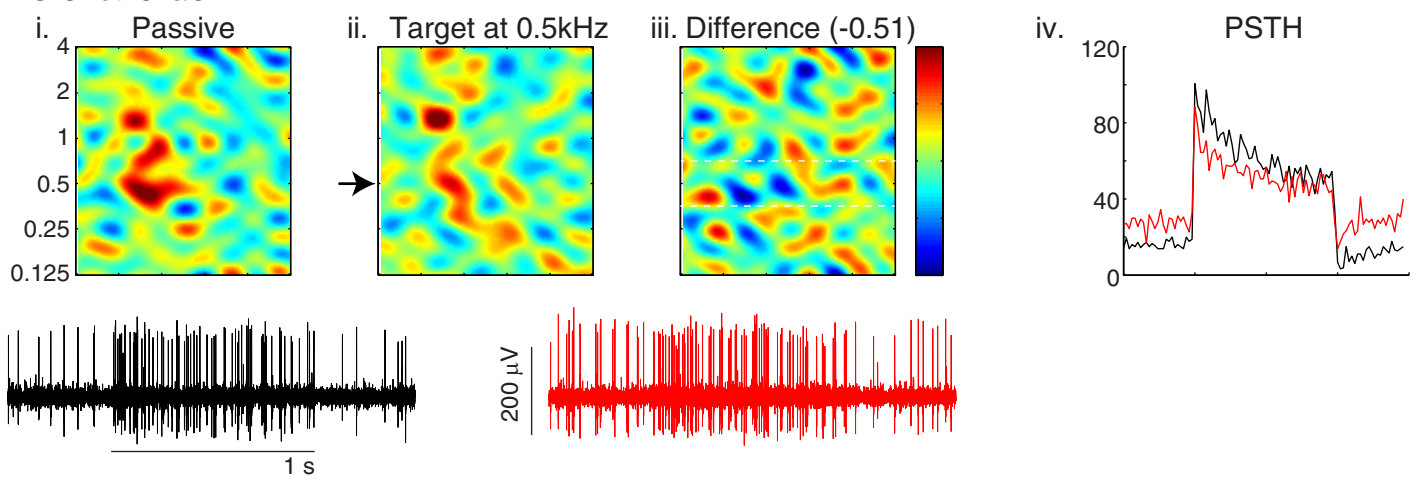

C ICC: mgc52eCh2

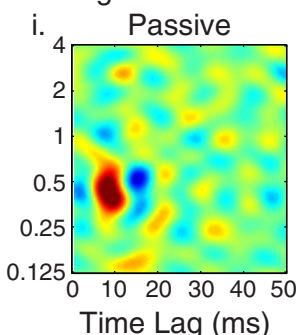

ii. Target at $0.6 \mathrm{kHz}$

iii. Difference (-0.17)
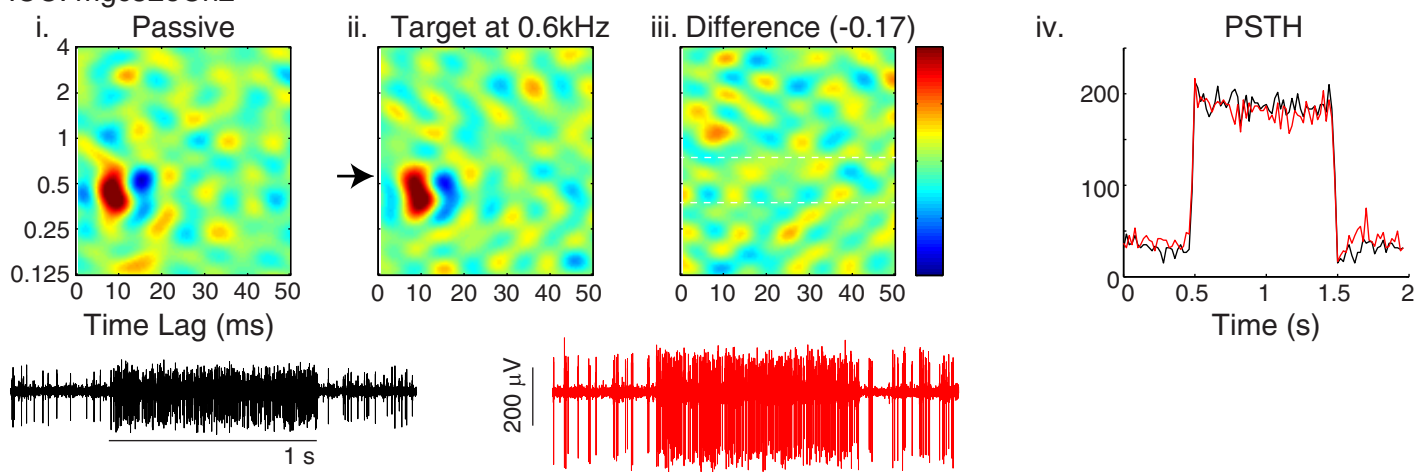

Figure 3. Examples of task-related plasticity in the IC. A, STRF of an ICC neuron estimated by reverse correlation with the reference noise sounds in the passive (i) and active (ii) behavioral condition. The STRF heatmap indicates the stimulus frequencies and time lags correlated with excited (red) or suppressed (blue) neural spike rate. STRFs have been interpolated for display purposes only. The arrow indicates target frequency. The difference between active and passive STRFs (iii) shows slight suppression near the target frequency. Local STRF changes were measured in a one-octave band (white dotted lines) centered at the target frequency. The PSTH (iv), computed by averaging across all reference TORC responses, is suppressed in the active (red) condition. The raw waveform of the spike responses to one reference stimulus in the passive (black) and active (red) conditions is shown at the bottom. The black bar indicates the duration of the $1 \mathrm{~s} s t i m u l u s . B$, Same format as $\boldsymbol{A}$ for an $\mathrm{NCIC}$ neuron with a suppressed receptive field during behavior. C, Same format as $\boldsymbol{A}$ for an ICC neuron with no receptive field change in the active condition. In this recording, the raw spike waveform (bottom) was larger during behavior but the unit isolation remained stable. norm. amp., normalized amplitude; sp/s, spikes/second.

tion task was then significantly suppressed at the target frequency (Fig. 3Aii). We quantified this effect by the local STRF change, defined as the fraction difference between the active and passive STRF at the target frequency $(-0.32$; Fig. 3Aiii). The peristimulus time histograms (PSTHs) show that the average driven response to the TORC stimuli for this neuron was also suppressed during behavior, while the spontaneous spike rate was unchanged (Fig. 3Aiv).

For an NCIC neuron (Fig. 3Bi), the STRF measured in the passive condition was more complicated than the ICC example, consistent with the more complex tuning properties typically reported for NCIC (Aitkin et al., 1975). The receptive field had a longer delay ( $\sim 15 \mathrm{~ms})$ and revealed two response peaks: a maximum near $0.5 \mathrm{kHz}$ and a side peak at $1.4 \mathrm{kHz}$. During behavior, the target was positioned on the BF. As in the previous example, the STRF was significantly suppressed at the target frequency but did not change significantly at the peak away from the target (Fig. 3Bii). The difference plot indicates a local STRF change of -0.51 (Fig. 3Biii). The average TORC response also shows suppression during behavior, and in this case the spontaneous rate was also enhanced (Fig. 3Biv).

Not all neurons underwent changes during behavior. The STRF of another ICC neuron remained stably tuned to frequencies of $\sim 0.5 \mathrm{kHz}$ during passive listening and behavior, and there was no change in the PSTH response (Fig. $3 C$ ).

To summarize the effects of task engagement on activity in each region of the IC, STRF difference plots were aligned to the 
A

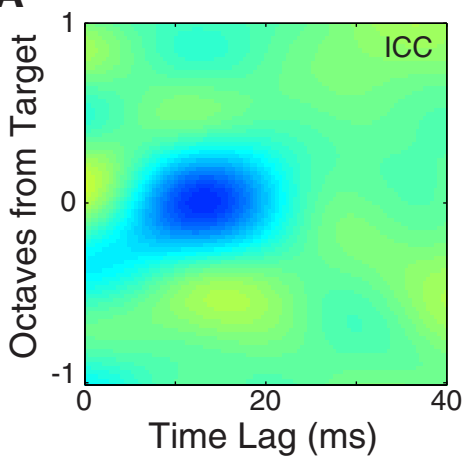

B

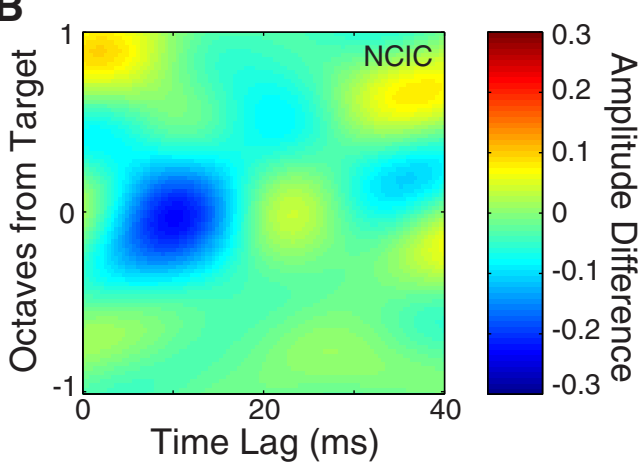

C

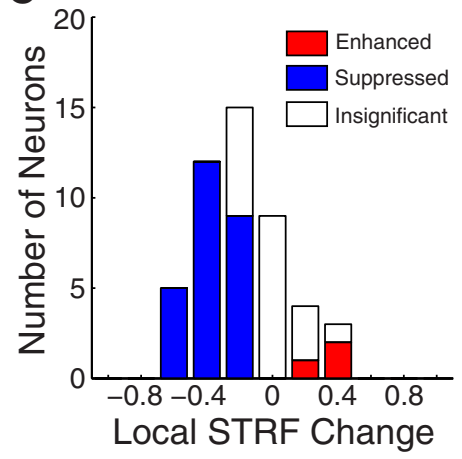

D

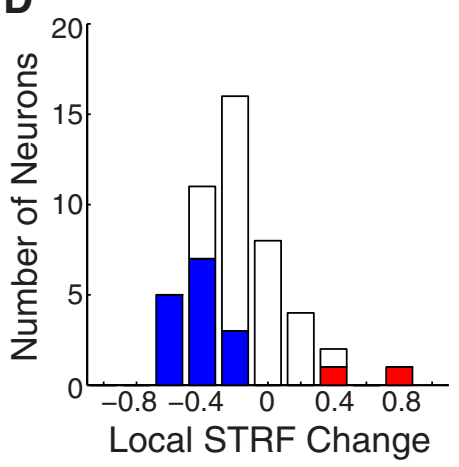

Figure 4. STRFs are suppressed at the target frequency during behavior. $\boldsymbol{A}$, Average STRF difference between active and passive behavioral conditions, aligned at the target frequency (all targets $<0.5$ octaves from $B F$ ) and averaged over all ICC neurons ( $N=$ 48). The blue region indicates average suppression around the target frequency. $\boldsymbol{B}$, Same format as $\boldsymbol{A}$ for all NCIC neurons $(N=47)$. C, Histogram plots the local STRF change (fraction difference between the behaving and passive STRF in a 1 octave band centered at the target frequency) in each ICC neuron. Filled bars indicate 29 neurons with significant changes $(p<0.05$, jackknifed $t$ test). $D$, Same format as $C$ for NCIC neurons. The median changes in ICC $(-0.32, p<1 \mathrm{e}-4$, signed-rank test $)$ and NCIC $(-0.42, p=$ 0.002 , signed-rank test) were not significantly different ( $p=0.27$, rank-sum test).

target frequency and averaged across neurons. For the 48 ICC neurons, the average STRF difference shows that responses were typically suppressed in the frequency region around the target (Fig. $4 A$ ). In the ICC sample, local STRF changes were significantly negative in 26 neurons and positive in 3 . The median local STRF change for those 29 neurons was -0.32 ( $p<1 \mathrm{e}-4$, signedrank test; Fig. 4C).

The pattern of local STRF changes was nearly identical for the 47 NCIC neurons. The average STRF difference also showed suppression near the target frequency (Fig. 4B). The average was slightly noisier than for ICC, likely reflecting the greater complexity of many STRFs measured in this region. In the NCIC sample, local STRF changes were significantly negative in 15 neurons and positive in 2 (Fig. $4 D$ ). The median local STRF change measured across all behaviorally modulated NCIC neurons was $-0.42 \%$ ( $p=0.002$, signed-rank test) and was not significantly different from changes in ICC ( $p=0.27$, rank-sum test).

These STRF changes demonstrate that, as in auditory cortical areas, IC neurons can alter their sensory selectivity when animals engage in an auditory detection task.

\section{Task-related plasticity has a global component}

The changes we observed in PSTH responses (Fig. 3A,B) suggested that the local STRF changes in IC neurons were accompanied by an overall change in responsiveness during behavior, i.e., global gain changes. In the visual system, gain changes are observed across the entire tuning curves of some cortical neurons, depending on the relationship between a neuron's spatial recep- tive field and the locus of spatial attention (McAdams and Maunsell, 1999). To test for global gain changes in an IC neuron, we computed the PSTH evoked by each TORC stimulus under each behavior condition. We then plotted the driven firing rates during each $5 \mathrm{~ms}$ time bin (black points) in the active condition against the rates in the same time bins during the passive condition (Fig. 5A). The rate estimates measured in the $5 \mathrm{~ms}$ time bins were noisy. Therefore, they were binned in groups of 50 before fitting a line (gray points; see Materials and Methods). For this neuron, the resulting scatter plot was well fit by a line with a slope of 0.81 and an offset of -4.7 spikes/s, consistent with global suppression during behavior (slope, $<1$ ). We define this slope as the gain and the fraction change from a unity slope $(-0.19)$ as the global gain change. A global gain change of zero corresponds to no global effect on the receptive field of the neuron during behavior. The gain can be interpreted as the optimal multiplicative scaling factor applied to the passive driven rates to approximate the active driven rates. The offset can be interpreted as the mean change in driven spike rate across all TORC stimuli. While some neurons showed significant global gain changes (Fig. 5A), others did not (Fig. 5B).

We measured global gain changes to assess task-related plasticity across the combined sample of NCIC and ICC neurons (Fig. 5C). Global gain changes were significantly negative in 46 neurons and positive in 7 . The median global gain change across these 53 neurons was -0.20 ( $p<1 \mathrm{e}-7$, signed-rank test). There was substantial overlap between neurons showing global gain changes and those showing local STRF changes. Of the 46 neurons with significant local STRF changes, 36 (78\%) also had significant global gain changes. Overall, we found that global gain change was a more sensitive measure of task-related plasticity than local STRF change. This was particularly evident in the NCIC dataset, where 27 neurons had significant global gain changes but only 17 had significant local STRF changes. These results demonstrate that, concomitant with local STRF changes at the target frequency, the overall excitability of IC neurons is also altered when animals engage in an auditory detection task.

We also measured changes in the offset (average evoked rate) across the sample of IC neurons (Fig. 5D). Offsets can be caused by local deviations from a purely global gain change. The offset was not significantly different from zero in 47 neurons, was positive in 15 neurons, and was negative in 33 neurons. Although the median offset was significant $(-3.2$ spikes/s or $-4 \%$ of the firing rate range, $p=0.029$, signed-rank test), offset effects were typically small compared with global gain changes.

Finally, we considered changes in the spontaneous rate (Fig. 6), which were subtracted in the gain analysis. Significant changes (gray points) during behavior were found in 58 of 95 individual IC neurons ( $p<0.05, t$ test). However, spontaneous rates were not systematically increased or decreased across the sample of 
neurons (median, $11 \%$ increase relative to passive; $p=0.51$, signed-rank test).

Both of these results led us to focus on global gain changes in the remainder of the study.

\section{Task-related plasticity in the IC can persist after behavior}

Previous studies in cortex have found that task-related plasticity sometimes persists in passive periods after the detection task (Fritz et al., 2003, 2010). To measure the recovery or persistence of task-related plasticity in the IC, we compared global gain changes in the active and postpassive conditions relative to the prepassive baseline. Prepassive and postpassive responses were measured immediately before and after the behavior, respectively. For the example neuron shown in Figure $7 A$, the global gain change was positive in the active condition ( 0.34 , gray). In the postpassive condition immediately following behavior, the global gain change rapidly recovered to an insignificant value near zero (0.04; Fig. 7A, black).

A second neuron showed a persistent global gain change (Fig. 7B). The response was suppressed both during behavior $(-0.28)$ and in the postpassive condition $(-0.33)$. Task-related global gain changes were found in 24 of 40 neurons with unit isolation that remained stable enough to measure activity in the postpassive condition. We measured complete recovery of global gain changes in 7 of 24 neurons and partial recovery in 4 of 24 . Persistent or further changes in global gain were found in the remaining 13 of 24 neurons (Fig. 7C). Thus, a substantial portion of IC neurons showed persistent global gain changes following behavior.

\section{Task-related plasticity can depend on target frequency}

We next considered whether the plasticity measured in the IC depended on the difference between the neuron's BF and the target frequency. To test this possibility, we compared global gain changes in the same neuron between two behavior conditions: one with the target frequencies near $\mathrm{BF}$ (On-BF) and one $\geq 1$ octave from BF (Off-BF). For one ICC neuron (Fig. $8 A$ ), the target was placed $\mathrm{On}-\mathrm{BF}(8 \mathrm{kHz})$ during the first behavior session, and the global gain change was significantly positive (Fig. $8 B$, red). When the target was subsequently placed three octaves below $\mathrm{BF}(1 \mathrm{kHz})$ for the next behavior session, the global gain change was even larger (blue). Thus, for this neuron, the global gain change depended on the distance between target frequency and BF. Another example neuron had a negative global gain change during a behavior session with an On-BF target and a positive global gain change during a behavior session with an Off-BF target (Fig. 8C).

Neural activity was measured in 27 IC neurons (seven with both above and below BF targets) during multiple behavior sessions with On-BF and Off-BF targets (Fig. 8D). In 20 of 34 cases,
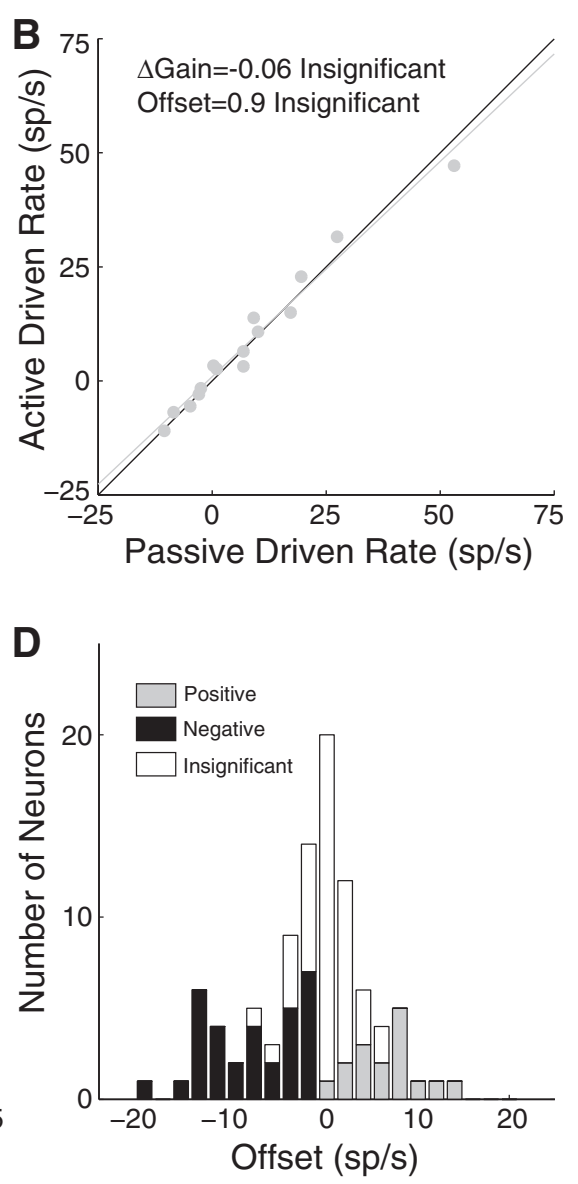

Figure 5. Task-related plasticity has a global component. $\boldsymbol{A}$, Scatter plot of driven firing rates (small black points) for each $5 \mathrm{~ms}$ bin during reference stimuli in active versus passive conditions for one neuron. A small amount of variability has been added to visualize overlapping points. Gray line plots the best linear fit to the binned rates (gray points). The global gain change $(-0.19=$ 作 Filled bars indicate the offsets that are significantly different from 0 (median, -3.2 spikes $/ \mathrm{s}$ or $-4 \%$ of the firing rate range, $p=$ 0.029 , signed-rank test). sp/s, spikes/second.

the global gain changes were not significant (7 of 20) or not significantly different between behavior sessions ( 13 of 20 ). However, in 13 of 14 of the remaining cases, the global gain change for On-BF targets was significantly negative when measured relative to Off-BF targets (median, $-0.23 ; p=0.002$, signed-rank test). Thus, for approximately half of the neurons showing behavior effects, global gain was suppressed for On-BF targets relative to Off-BF targets. Among these neurons, the size of suppression between On-BF and Off-BF conditions was indistinguishable from suppression between the On-BF active condition and the prepassive condition ( $p=0.88$, rank-sum test; Fig. $5 C$ ). These results demonstrate that the receptive field changes measured during behavior depend on the difference between the neuronal $\mathrm{BF}$ and the target frequency.

We also examined changes in spontaneous activity across behavioral blocks with different target frequencies (Fig. $8 E$ ). The spontaneous rate did not change during behavior (behaving vs passive) in 7 of 34 cases (black) and changed during behavior but did not depend on the target frequency in 15 of 34 cases (blue). However, in the remaining cases ( 12 of 34 ), the spontaneous rate was significantly increased or decreased between On-BF and Off-BF target behaviors (red). Thus, the spontaneous rate can be affected by the 


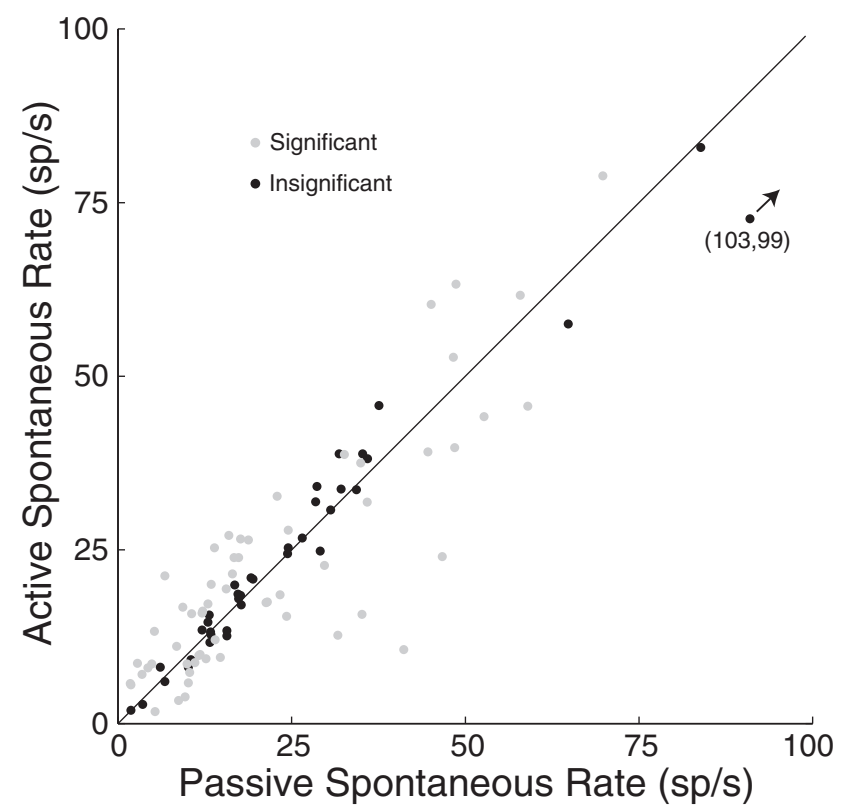

Figure 6. Spontaneous rate changes during behavior. Spontaneous rate during behaving versus passive conditions for each IC neuron. Significant changes during behavior (gray points, $p<0.05, t$ test) were found in 58 of $95 \mathrm{IC}$ neurons. However, rates were not systematically increased or decreased across neurons (median, $11 \%$ increase relative to passive; $p=0.51$, signed-rank test). sp/s, spikes/second.

target frequency but the magnitude of the change is often smaller than the active-passive difference (compare with Fig. 6).

\section{Comparison of task-related plasticity between the IC and the auditory cortex}

Suppressive local STRF changes were previously reported in A1 during the same detection task (David et al., 2012). By using an identical behavior in the current study, we were able to directly compare task-related plasticity between A1 and the IC. We reanalyzed local STRF changes for data from both areas during behaviors with On-BF targets and for a narrow (onethird octave) spectral window around the target frequency, as was used previously in A1. We found similar effects in both areas, with negative local STRF changes being dominant. In the IC, local STRF changes in 34 neurons were negative and 7 were positive (median, $-0.47 ; p<1 \mathrm{e}-10$, signed-rank test; Fig. 9A). In A1, local STRF changes in 36 units were negative and 12 were positive (median, $-0.38 ; p<1 \mathrm{e}-3$, signed-rank test; Fig. $9 B$ ). The median local STRF changes were not significantly different between areas $(p=0.4$, rank-sum test).

Although the local STRF changes were similar, the distributions of global gain changes were clearly different between the IC (Fig. 9C) and A1 (Fig. 9D). Nearly all the global gain changes were negative in the IC (median, $-0.20 ; p<1 \mathrm{e}-7$, signed-rank test), but they were far more heterogeneous in A1. Global gain changes were negative in 24 of $108 \mathrm{~A} 1$ units and positive in 31 of 108 . The median global gain change in A1 (0.23) was not significantly different from zero $(p=0.42$, signed-rank test) but was significantly different from the IC ( $p<1 \mathrm{e}-3$, rank-sum test). The range of global gain changes was also greater in A1, where several units had very large changes of $>0.5$. Thus, while overall driven activity is suppressed in the IC during the tone detection behavior, compensatory increases and decreases in global gain maintain approximately the same overall driven activity level in A1.
A

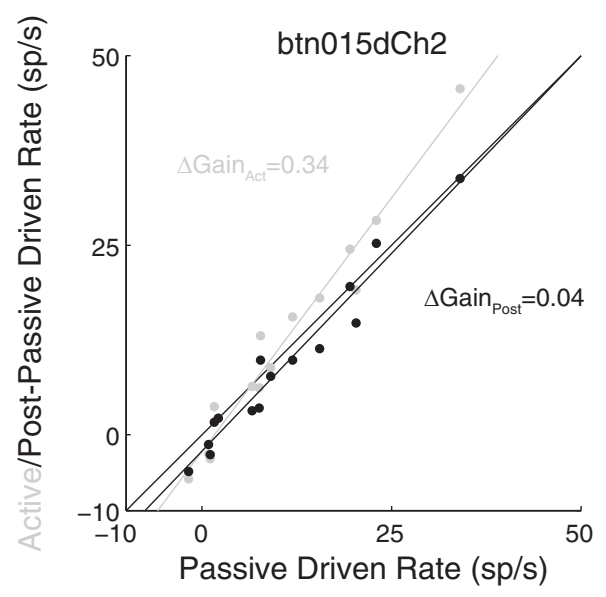

B

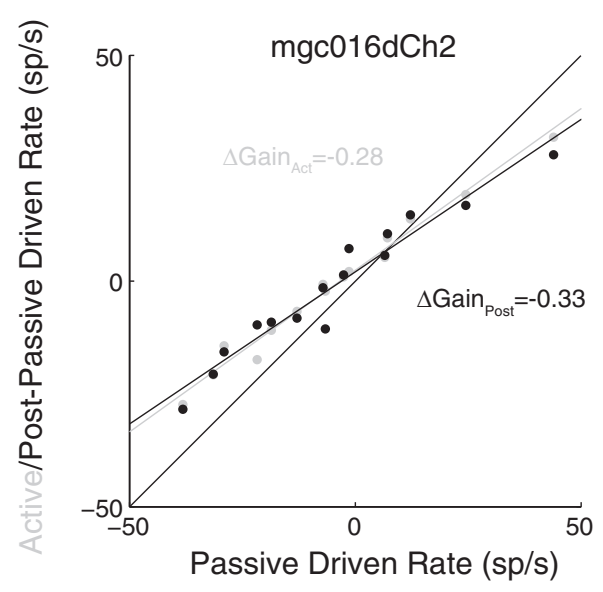

C

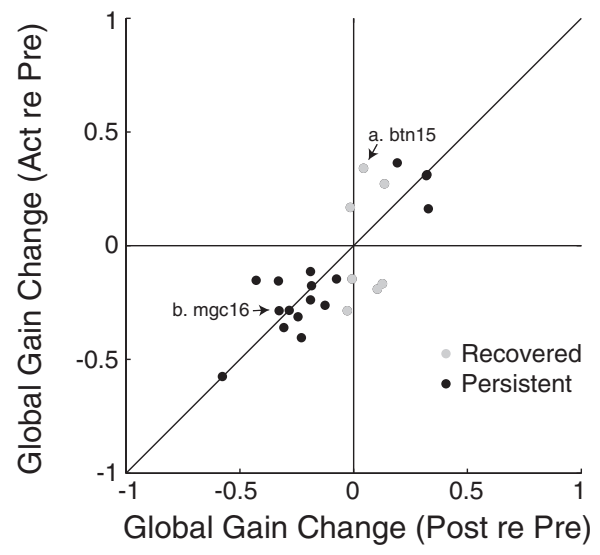

Figure 7. Recovery and persistence of task-related plasticity. A, Active (gray) and postpassive (black) driven firing rate versus prepassive driven firing rates for an ICC neuron. The global gain change is positive (slope, $>1$ ) and recovers in the postpassive condition immediately following the behavior (global gain change, 0 ; postpassive relative to prepassive). $\boldsymbol{B}$, Same format as $\boldsymbol{A}$ for a different IC $($ neuron with a negative global gain change that persists in the postpassive condition. $C$, Global gain changes relative to prepassive in the active (Act re Pre) versus postpassive (Post re Pre) condition in 24 of 40 neurons with significant changes during behavior. The global gain change recovered completely in 7 neurons (gray) and persisted or only partially recovered in the remaining 17 neurons (black). Arrows indicate the examples in $\boldsymbol{A}$ and $\boldsymbol{B}$. sp/s, spikes/second. 


\section{Opposing local tuning and global gain changes are more common in $\mathrm{Al}$}

To understand the interaction between local tuning and global gain changes in the IC and A1, we computed the fraction of the local STRF change accounted for by the global gain change. To compensate for global gain changes, we scaled the passive STRF by the gain before computing the active-passive difference. Residual changes in the STRF difference were defined as a local tuning change (David et al., 2008). In an example IC neuron, the global gain change (Fig. 10A, bottom, left) fully accounted for the local STRF change (Fig. 10A, top), and the local tuning change (Fig. 10A, bottom, right) was not significant. Thus, in this case the local STRF change was simply the result of a behaviorally driven global gain change and no local tuning change.

In $\mathrm{A} 1$, the interaction between global gain and local tuning changes was more complex. For an example A1 neuron, the local STRF change was not significant (Fig. $10 B$, top). However, the global gain change was significantly positive (Fig. 10B, bottom, left). When the passive STRF was scaled by the gain, a small but significant local tuning change was unmasked (Fig. 10B, bottom, right). Thus, for this $\mathrm{A} 1$ neuron, the global and local changes in the receptive field had opposite signs and canceled out at the target frequency.

We measured local tuning changes across the sample of IC and Al units after scaling the passive STRF to remove the effects of global gain changes (Fig. $10 C, D)$. In both areas, fewer units had significant local tuning changes than local STRF changes (IC: 22 vs 41 ; A1: 35 vs 48 ). Thus, for approximately half of the IC neurons and one-quarter of the A1 units, local STRF changes were a reflection of global gain changes during behavior. For the units with local tuning changes, the median change in A1 (-0.56) was significantly larger than the local tuning change in the IC $(-0.28 ; p=0.035$, rank-sum test).

Comparing local tuning changes and global gain changes within units revealed a qualitative difference in behavior effects between the IC and A1 (Fig. 10C,D). In both areas, negative global gain changes were often accompanied by negative local tuning changes (Fig. 1C, model). However, units with positive global gain changes followed different patterns. In the IC, positive global gain changes were rare and co-occurred with local tuning changes in only three neurons. In A1, positive global gain changes were much more common and were usually associated with negative local tuning changes ( 24 of 35 units; Fig. 10D, lower right quadrant). This class of units with
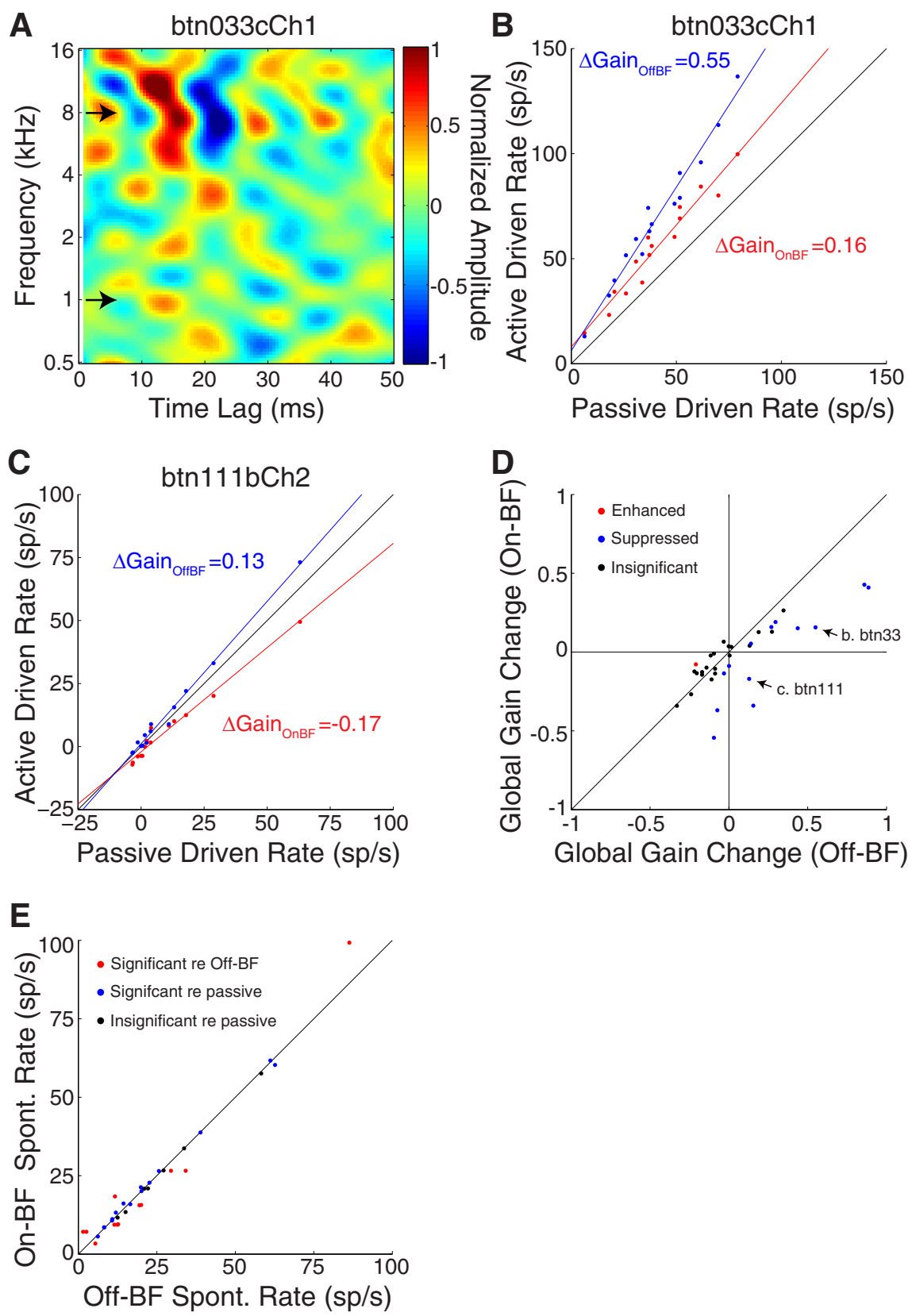

Figure 8. Task-related plasticity depends on target frequency. $A, S T R F$ from an ICC neuron showing the frequencies (arrows) of On-BF ( $8 \mathrm{kHz})$ and $0 \mathrm{ff}-\mathrm{BF}(1 \mathrm{kHz})$ targets for two consecutive behavior sessions. $\boldsymbol{B}$, Driven firing rates measured in active condition for the $0 \mathrm{n}-\mathrm{BF}$ target (red) and Off-BF target (blue) versus prepassive driven rates for the neuron in $\boldsymbol{A}$. The global gain change is positive for the On-BF target and further enhanced for the Off-BF target. C, On-BF versus Off-BF gain comparison for a different ICC neuron, plotted as in $\boldsymbol{B}$. Relative to passive, the global gain change is negative for the $0 \mathrm{n}-\mathrm{BF}$ target and positive for the Off-BF target. $D$, Scatter plot of the global gain changes for $0 \mathrm{n}-\mathrm{BF}$ targets versus Off-BF targets (1-3 octaves above or below BF). In 20 of 34 cases, responses did not change significantly during behavior and/or between targets (black). In 13 of 14 of the remainder, global gain was suppressed (blue) for $0 \mathrm{n}-\mathrm{BF}$ relative to $0 \mathrm{ff}-\mathrm{BF}$ and the median difference was significant $(-0.23, p=0.002$, signed-rank test). Arrows indicate the example neurons in $\boldsymbol{B}$ and $\boldsymbol{C}$. $\boldsymbol{E}$, Spontaneous (spont.) rate during behavior sessions with On-BF versus Off-BF targets. Colors indicate spontaneous rates that did not change during behavior (behaving vs passive, black), that changed during behavior but did not depend on the target frequency (blue), or that changed significantly between On-BF and Off-BF target behaviors (red). sp/s, spikes/second.

global and local changes of opposite sign (Fig. 1D, model) emerges in the processing stages between the IC and A1.

\section{Discussion}

We found that the spectrotemporal selectivity in the IC undergoes rapid plasticity as ferrets engage in an auditory detection task. Responses during behavior sessions with target tones 

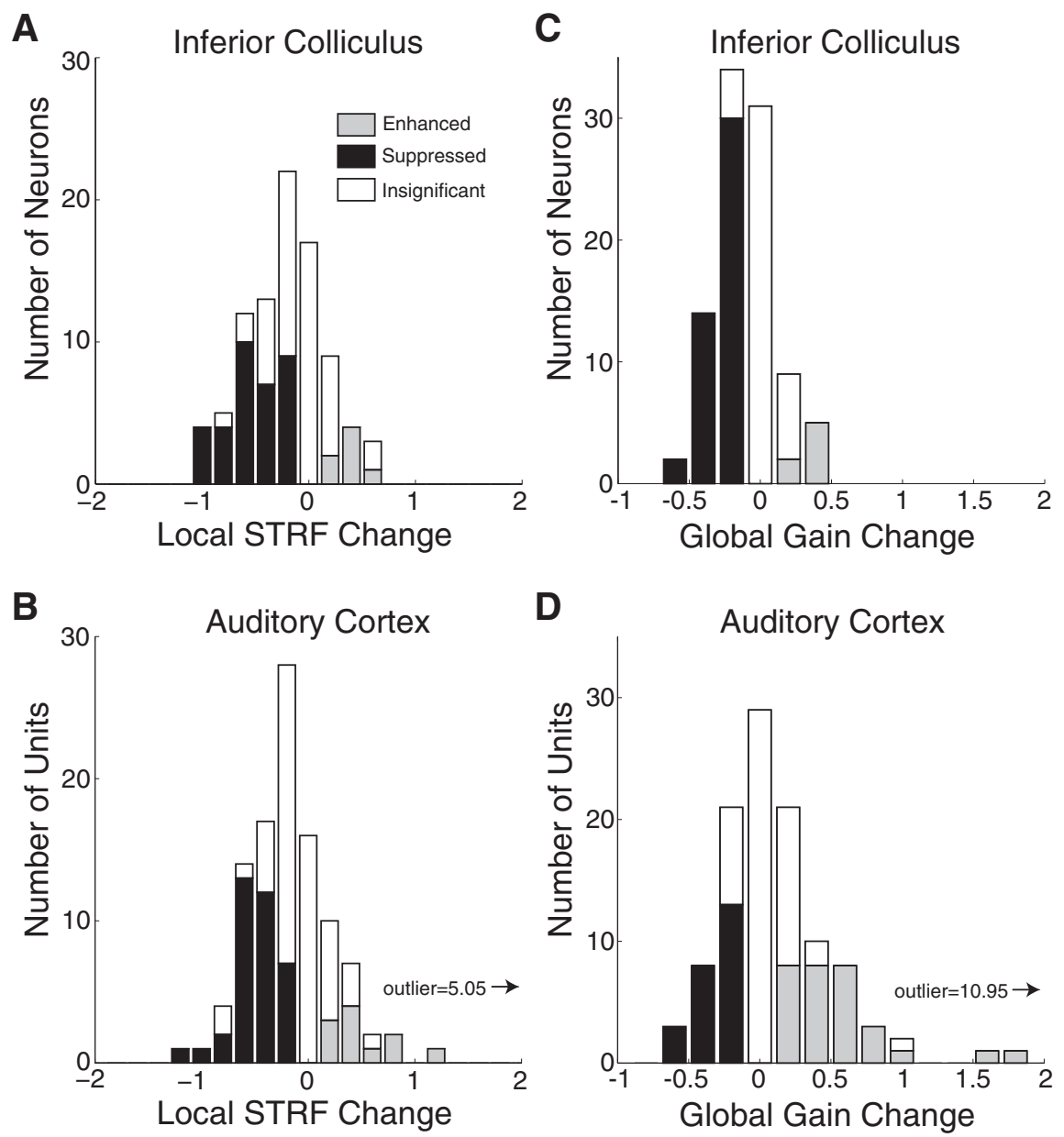

Figure 9. Comparison of task-related plasticity between the IC and A1. A, Histogram plots the local STRF change in each IC neuron. (IC data from Fig. 4 reanalyzed using a $1 / 30$ octave band to match the $A 1$ analysis published previously). Filled bars indicate 41 neurons showing significant changes ( $p<0.05$, jackknifed $t$ test). $\boldsymbol{B}$, Same format as $\boldsymbol{A}$ for 108 units in A1. The dataset contains a combination of single neurons and multiunits (see Materials and Methods). Filled bars indicate 48 units with significant changes. Arrow indicates one outlier with a very large local STRF change. The median local STRF changes in the IC $(-0.47, p<1 \mathrm{e}-10)$ and $\mathrm{A} 1(-0.38, p<1 \mathrm{e}-3)$ were not significantly different $(p=0.40$, rank-sum test). $C$, Histogram plots the global gain change in each IC neuron (data from Fig. 5 C but with $2 \times$ wider bins). Filled bars indicate significant changes $(p<0.05$, jackknifed $t$ test). D, Same as $C$ for the $108 \mathrm{~A} 1$ units. Note the large number of units with positive global gain changes (gray). The arrow indicates one outlier with a very large global gain change. The median global gain changes in the IC $(-0.20, p<1 \mathrm{e}-7)$ and $\mathrm{A} 1(0.23, p=0.42)$ were significantly different ( $p<1 \mathrm{e}-3$, ranked-sum test).

matched to the neuronal $\mathrm{BF}(\mathrm{On}-\mathrm{BF})$ were suppressed in approximately half of the neurons studied. The suppression resulted from a combination of local tuning changes and global gain changes. This task-related plasticity was also frequency dependent: responses during behavior sessions with Off-BF targets were less suppressed or even enhanced relative to the passive state. Previous studies have argued that task-related plasticity in the cortex reflects attention to auditory stimulus features (Fritz et al., 2003, 2010; David et al., 2012; Niwa et al., 2013; Atiani et al., 2014). Under this assumption, our results provide direct support for the inclusion of the IC in hierarchical models of selective auditory attention that have previously been exclusive to the cortex.

The task-related plasticity observed in the IC had many similarities with plasticity in $\mathrm{A} 1$ measured under the same experimental conditions (David et al., 2012). The magnitude of STRF suppression near the target frequency was indistinguishable between areas. The changes were frequency dependent, could have both a local and global component, and persisted in some neu- rons while recovering in others. Unique to the IC, both local tuning and global gain changes tended to be suppressive, while in A1, global gain changes were equally likely to be enhanced or suppressed. This suggests that some features of task-related plasticity found in A1 are already present in the IC, but other properties emerge at later processing stages.

\section{Arousal versus task-specific effects}

Can the plasticity in IC be attributed simply to changes in arousal state during task engagement? For the purpose of discussion, we define the level of arousal (e.g., asleep, passive, engaged) as a brain state that is influenced by general task engagement (not specific to auditory behavior). We found that receptive field changes in IC persisted in periods immediately following behavior more often than they recovered. One interpretation of this result is that the level of arousal increased during the behavior, causing suppression (Otazu et al., 2009), and remained high after the behavior was completed. However, two features of the task-related plasticity are difficult to reconcile with this interpretation. First, only sound-evoked activity in IC was systematically suppressed. Spontaneous rates changed in both directions during behavior (Fig. 6). Second, responses were more suppressed during On-BF than Off-BF behaviors, conditions during which the level of arousal was similar. Thus a component of the plasticity is tuned to the target frequency and independent of the level of arousal.

Another interpretation of the persistent plasticity is that it reflects a taskrelated memory trace (Bakin and Weinberger, 1990). Maintaining a brain state with improved discrimination could be beneficial to the animal in subsequent behavioral sessions until changing behavior conditions require performing a different discrimination. Indeed, persistence may be a necessary step in perceptual learning (Yang and Maunsell, 2004). Further characterization of the time course of these persistent effects in the IC will be an important direction for future studies.

\section{Comparison with previous studies}

The task-related plasticity we observed in the IC was largely consistent with effects previously reported in Al for the same task (David et al., 2012). There were, however, some striking differences. Most prominently, behavioral effects were much more likely to persist in the IC than in A1 (Fritz et al., 2003) and global gain changes were largely suppressive in the IC versus both suppressive and facilitative in A1. In addition, behavioral effects in the ferret are larger outside of A1 (Atiani et al., 2014). Given that these cortical regions project to NCIC but not ICC (Bajo et al., 2007), it might be expected that task-related plasticity would also be larger in NCIC compared with ICC. However, we found effects 
A

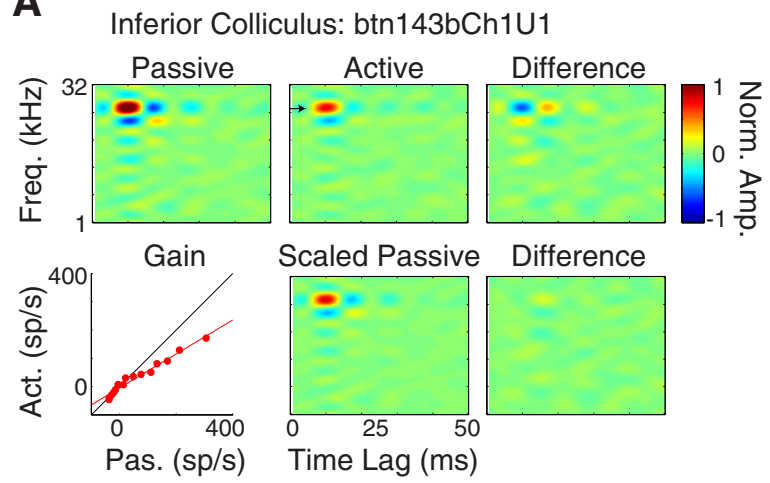

B

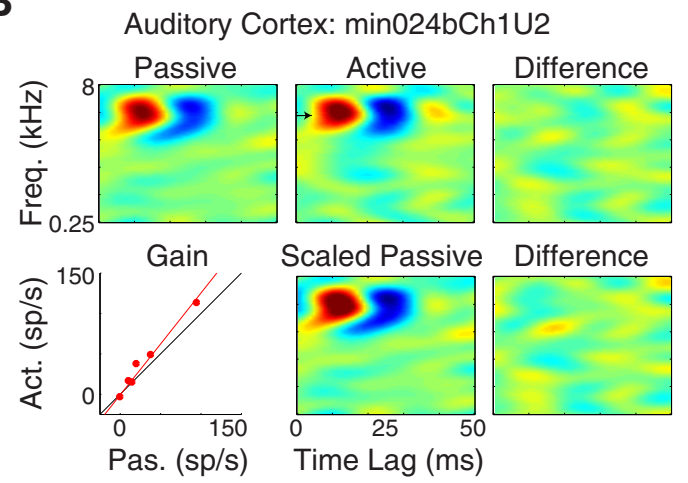

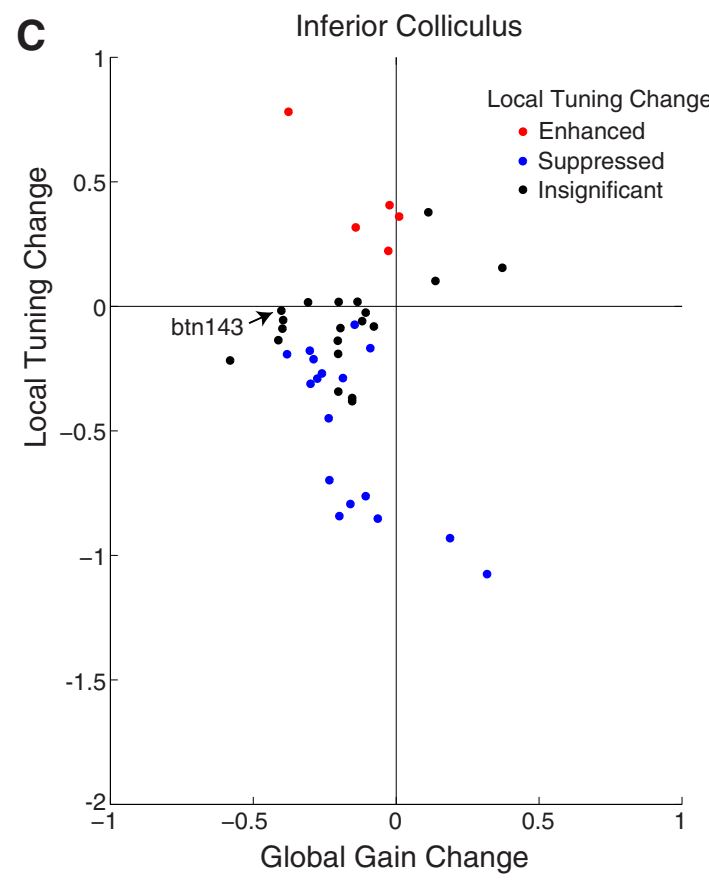

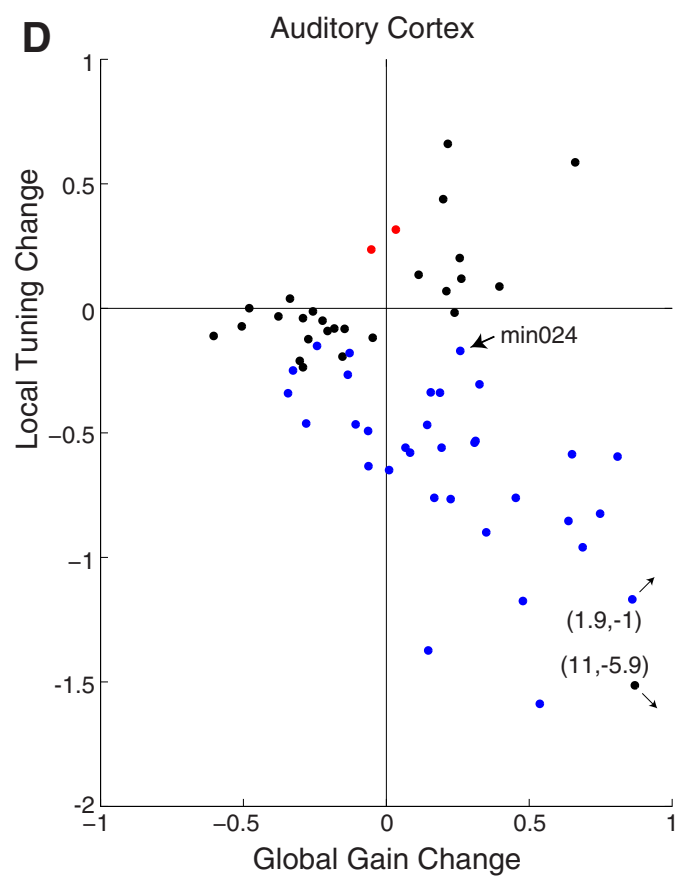

Figure 10. Opposing global gain and local tuning changes emerge between the IC and A1. A, Top, Negative local STRF change $(-0.42)$ measured during behavior for an example IC neuron (same format as Fig. 3A). Bottom left, Negative global gain change ( -0.40$)$. Bottom middle, Passive STRF scaled by the gain. After rescaling of the passive STRF, the local STRF change is eliminated (i.e., no local tuning change; bottom right). $\boldsymbol{B}$, Same format as $\boldsymbol{A}$ for an A1 neuron. The local STRF change ( 0.09$)$ is not significant but the global gain change is positive ( 0.26$)$. Scaling the passive STRF by the gain reveals significant suppression at the target [i.e., a local tuning change (-0.17); bottom right]. C, Local tuning change versus global gain change in the IC. D, Same format as $($ for A1. Unlike the IC, 24 of $35 \mathrm{~A} 1$ units with negative local tuning changes have positive global gain changes (fourth quadrant). The median local tuning change in $\mathrm{A} 1$ ( -0.56 ) is significantly larger than in the IC $(-0.28, p=0.035$, rank-sum test). Act., active firing rate; Pas., passive firing rate; Freq., frequency; sp/s, spikes/second.

of similar magnitude in both regions. These differences may reflect a role of the auditory cortex in coding higher-level behavioral signals (Brosch et al., 2005; Bizley et al., 2013) and a role of the IC that is specific to auditory processing.

A previous study in macaque monkeys compared IC activity during performance of a simple reaction-time task and passive listening (Ryan and Miller, 1977). The authors found increases in both driven rate and latency, which differs from our results. In addition, the authors report no change in spontaneous rates while we found changes between behaving and passive as well as between behavior sessions with different target frequencies (Figs. $6,8 E)$. The cause for these discrepancies is unclear but several factors could explain the different results. The previous study used only target sounds and included no distractor (reference) sounds. This difference in task structure, which can strongly affect plasticity (David et al., 2012), may explain the discrepancies among studies. The discrepancies may also result from the differences in the corticocollicular system between monkeys and other mammals (Winer, 2005). Finally, the macaque study did find that responses recovered in some neurons but persisted in others, which is consistent with our results.

\section{Mechanisms of task-related plasticity}

In the mammalian auditory system, dense corticocollicular projections send excitatory feedback from the cortex to the IC (Winer, 2005). The existence of this large pathway suggests that the ongoing activity in the auditory cortex can rapidly and dynamically modulate responses in the IC. Consistent with this hypothesis, previous studies using cortical inactivation (Zhang et al., 1997) and electrical stimulation (Ma and Suga, 2001) have produced spectral tuning changes in the IC. The target-specific suppression we measured in the IC is very similar to suppression in A1. If ongoing activity in A1 is suppressed, the IC could also be suppressed through a decrease in positive feedback. The evidence that a subset of the corticocollicular projections to the IC are 
tonotopic (Winer, 2005; Bajo et al., 2007) and excitatory (Ito and Oliver, 2010) further supports this idea.

However, one of our experimental findings is more difficult to reconcile with a purely corticocollicular mechanism. The projection from the cortex to the NCIC (the lateral and dorsal divisions) is denser and often ends in larger synaptic boutons than the projection to the ICC (Bajo et al., 2007). This larger anatomical projection suggests that task-related plasticity should be greater in the NCIC. However, we found that the magnitude of the plasticity was similar in these two regions.

In addition to the corticocollicular system, neuromodulatory systems intrinsic to the midbrain could also alter neural receptive fields. The IC receives input from nuclei that release serotonin, dopamine, acetylcholine, and norepinephrine (Hurley and Sullivan, 2012). Many of these modulatory nuclei themselves receive direct connections from the cortex and their level of activation likely depends on behavioral context. In vivo iontophoretic studies have demonstrated that neuromodulators can affect global and/or local tuning in a subset of IC neurons (Habbicht and Vater, 1996; Hurley and Pollack, 2001; Gittelman et al., 2013). Thus the possibility that task-related plasticity is first generated in the IC by indirect cortical activation of modulatory systems cannot, at present, be ruled out.

Some components of task-related plasticity might also be inherited from the auditory brainstem or the cochlea. Although the densest corticofugal projections target the IC, numerous smaller projections synapse in the auditory brainstem (Winer, 2006). Modulatory systems that project to the brainstem could also provide a pathway for behavioral changes that are inherited by the IC (Schofield et al., 2011). One previous study in the cochlear nucleus reported that single-neuron responses were altered during behavior (Ryan et al., 1984), while another found no effect (May and Sachs, 1992). However, the temporal tasks used in these studies cannot be directly compared with the spectral task used here. A separate study found that switching attention from an auditory to a visual task can reduce cochlear sensitivity (Delano et al., 2007), presumably through activation of the olivocochlear feedback system (Galambos, 1956). The contribution of brainstem and peripheral mechanisms to the task-related plasticity at the level of the IC is currently unknown.

Thus several questions about the function and mechanisms of task-related plasticity in the auditory subcortical network remain to be answered. Many pathways provide behavioral signals to the IC, but it is unclear which ones dominate in different behavioral contexts. Whether attention to the spectral, spatial, and temporal features of sounds affects their representation in a unique or general way will provide useful information to constrain models of task-related plasticity. Also, although the thalamus can undergo changes when animals engage in behavior (Jaramillo et al., 2014), its role in transforming task-related changes between the IC and A1 is unclear. Further exploration of how different behavioral contexts affect the subcortical auditory system will resolve these questions.

\section{References}

Aitkin LM, Webster WR, Veale JL, Crosby DC (1975) Inferior colliculus. I. Comparison of response properties of neurons in central, pericentral, and external nuclei of adult cat. J Neurophysiol 38:1196-1207. Medline

Atiani S, David SV, Elgueda D, Locastro M, Radtke-Schuller S, Shamma SA, Fritz JB (2014) Emergent selectivity for task-relevant stimuli in higherorder auditory cortex. Neuron 82:486-499. CrossRef Medline

Bajo VM, Nodal FR, Bizley JK, Moore DR, King AJ (2007) The ferret auditory cortex: descending projections to the inferior colliculus. Cereb Cortex 17:475-491. Medline
Bajo VM, Nodal FR, Moore DR, King AJ (2010) The descending corticocollicular pathway mediates learning-induced auditory plasticity. Nat Neurosci 13:253-260. Medline

Bakin JS, Weinberger NM (1990) Classical conditioning induces CSspecific receptive field plasticity in the auditory cortex of the guinea pig. Brain Res 536:271-286. CrossRef Medline

Bizley JK, Cohen YE (2013) The what, where and how of auditory-object perception. Nat Rev Neurosci 14:693-707. CrossRef Medline

Bizley JK, Walker KM, Nodal FR, King AJ, Schnupp JW (2013) Auditory cortex represents both pitch judgments and the corresponding acoustic cues. Curr Biol 23:620-625. CrossRef Medline

Brosch M, Selezneva E, Scheich H (2005) Nonauditory events of a behavioral procedure activate auditory cortex of highly trained monkeys. J Neurosci 25:6797-6806. CrossRef Medline

Cherry EC (1953) Some experiments on the recognition of speech, with one and two ears. J Acoust Soc Am 25:975-979. CrossRef

David SV, Hayden BY, Mazer JA, Gallant JL (2008) Attention to stimulus features shifts spectral tuning of V4 neurons during natural vision. Neuron 59:509-521. CrossRef Medline

David SV, Mesgarani N, Fritz JB, Shamma SA (2009) Rapid synaptic depression explains nonlinear modulation of spectro-temporal tuning in primary auditory cortex by natural stimuli. J Neurosci 29:3374-3386. CrossRef Medline

David SV, Fritz JB, Shamma SA (2012) Task reward structure shapes rapid receptive field plasticity in auditory cortex. Proc Natl Acad Sci U S A 109:2144-2149. CrossRef Medline

Delano PH, Elgueda D, Hamame CM, Robles L (2007) Selective attention to visual stimuli reduces cochlear sensitivity in chinchillas. J Neurosci 27: 4146-4153. CrossRef Medline

DiCarlo JJ, Lane JW, Hsiao SS, Johnson KO (1996) Marking microelectrode penetrations with fluorescent dyes. J Neurosci Methods 64:75-81. CrossRef Medline

Ding N, Simon JZ (2012) Emergence of neural encoding of auditory objects while listening to competing speakers. Proc Natl Acad Sci U S A 109: 11854-11859. CrossRef Medline

Efron B, Tibshirani RJ (1998) Introduction to the bootstrap. Boca Raton, FL: CRC.

Fritz J, Shamma S, Elhilali M, Klein D (2003) Rapid task-related plasticity of spectrotemporal receptive fields in primary auditory cortex. Nat Neurosci 6:1216-1223. CrossRef Medline

Fritz JB, David SV, Radtke-Schuller S, Yin P, Shamma SA (2010) Adaptive, behaviorally gated, persistent encoding of task-relevant auditory information in ferret frontal cortex. Nat Neurosci 13:1011-1019. CrossRef Medline

Galambos R (1956) Suppression of auditory nerve activity by stimulation of efferent fibers to cochlea. J Neurophysiol 19:424-437. Medline

Gittelman JX, Perkel DJ, Portfors CV (2013) Dopamine modulates auditory responses in the inferior colliculus in a heterogeneous manner. J Assoc Res Otolaryngol 14:719-729. CrossRef Medline

Habbicht H, Vater M (1996) A microiontophoretic study of acetylcholine effects in the inferior colliculus of horseshoe bats: implications for a modulatory role. Brain Res 724:169-179. CrossRef Medline

Hurley LM, Pollak GD (2001) Serotonin effects on frequency tuning of inferior colliculus neurons. J Neurophysiol 85:828-842. Medline

Hurley LM, Sullivan MR (2012) From behavioral context to receptors: serotonergic modulatory pathways in the IC. Front Neural Circuits 6:58. CrossRef Medline

Ito T, Oliver DL (2010) Origins of glutamatergic terminals in the inferior colliculus identified by retrograde transport and expression of VGLUT1 and VGLUT2 genes. Front Neuroanat 4:135. CrossRef Medline

Jaramillo S, Borges K, Zador AM (2014) Auditory thalamus and auditory cortex are equally modulated by context during flexible categorization of sounds. J Neurosci 34:5291-5301. CrossRef Medline

Klein DJ, Depireux DA, Simon JZ, Shamma SA (2000) Robust spectrotemporal reverse correlation for the auditory system: optimizing stimulus design. J Comput Neurosci 9:85-111. CrossRef Medline

Lauer AM, Slee SJ, May BJ (2011) Acoustic basis of directional acuity in laboratory mice. J Assoc Res Otolaryngol 12:633-645. CrossRef Medline

Lee CC, Middlebrooks JC (2011) Auditory cortex spatial sensitivity sharpens during task performance. Nat Neurosci 14:108-114. CrossRef Medline

Ma X, Suga N (2001) Plasticity of bat's central auditory system evoked by 
focal electric stimulation of auditory and/or somatosensory cortices. J Neurophysiol 85:1078-1087. Medline

May BJ, Sachs MB (1992) Dynamic range of neural rate responses in the ventral cochlear nucleus of awake cats. J Neurophysiol 68:1589-1602. Medline

McAdams CJ, Maunsell JH (1999) Effects of attention on orientationtuning functions of single neurons in macaque cortical area V4. J Neurosci 19:431-441. Medline

Mesgarani N, Chang EF (2012) Selective cortical representation of attended speaker in multi-talker speech perception. Nature 485:233-236. CrossRef Medline

Nakamoto KT, Jones SJ, Palmer AR (2008) Descending projections from auditory cortex modulate sensitivity in the midbrain to cues for spatial position. J Neurophysiol 99:2347-2356. CrossRef Medline

Niwa M, Johnson JS, O'Connor KN, Sutter ML (2012) Active engagement improves primary auditory cortical neurons' ability to discriminate temporal modulation. J Neurosci 32:9323-9334. CrossRef Medline

Niwa M, Johnson JS, O'Connor KN, Sutter ML (2013) Differences between primary auditory cortex and auditory belt related to encoding and choice for AM sounds. J Neurosci 33:8378-8395. CrossRef Medline

Otazu GH, Tai LH, Yang Y, Zador AM (2009) Engaging in an auditory task suppresses responses in auditory cortex. Nat Neurosci 12:646-654. CrossRef Medline

Rodgers CC, DeWeese MR (2014) Neural correlates of task switching in prefrontal cortex and primary auditory cortex in a novel stimulus selection task for rodents. Neuron 82:1157-1170. CrossRef Medline
Ryan A, Miller J (1977) Effects of behavioral performance on single-unit firing patterns in inferior colliculus of rhesus-monkey. J Neurophysiol 40:943-956. Medline

Ryan AF, Miller JM, Pfingst BE, Martin GK (1984) Effects of reaction time performance on single-unit activity in the central auditory pathway of the rhesus macaque. J Neurosci 4:298-308. Medline

Schofield BR, Motts SD, Mellott JG (2011) Cholinergic cells of the pontomesencephalic tegmentum: connections with auditory structures from cochlear nucleus to cortex. Hear Res 279:85-95. CrossRef Medline

Shinn-Cunningham BG (2008) Object-based auditory and visual attention. Trends Cogn Sci 12:182-186. CrossRef Medline

Slee SJ, Young ED (2013) Linear processing of interaural level difference underlies spatial tuning in the nucleus of the brachium of the inferior colliculus. J Neurosci 33:3891-3904. CrossRef Medline

Winer JA (2005) Three systems of descending projections to the inferior colliculus. In: Inferior colliculus (Winder JA, Schreiner Cem eds), pp 231-247. New York: Springer.

Winer JA (2006) Decoding the auditory corticofugal systems. Hear Res 212: $1-8$. CrossRef Medline

Yang T, Maunsell JH (2004) The effect of perceptual learning on neuronal responses in monkey visual area V4. J Neurosci 24:1617-1626. CrossRef Medline

Zhang Y, Suga N, Yan J (1997) Corticofugal modulation of frequency processing in bat auditory system. Nature 387:900-903. CrossRef Medline 